\title{
Evolution of protoplanetary disks
}

\author{
Cornelis P. Dullemond ${ }^{1}$ \\ ${ }^{1}$ Max-Planck-Institut für Astronomie \\ Königstuhl 17, 69117, Heidelberg, Germany \\ email: dullemon@mpia.de
}

In this review I gave an overview of the structure and evolution of protoplanetary disks, and how the evolution of dust affects this. This is an important topic because these determine the conditions under which planets are formed, or were formed in our solar system 4.5 billion years ago.

Nearly all protoplanetary disks display either dust features in emission or no dust features at all. This is strong evidence that the shape of these disks is flaring, i.e. the surface of the disk has a "bowl shape" such that the light of the star can illuminate the disk and create a warm surface layer on top of it (e.g. Chiang \& Goldreich (1997)). This irradiation process is the main source of heating in most parts of the disk, except perhaps at relatively early stages and in the regions relatively close to the star. In those regions the midplane temperature can be dominated by heating via the dissipation of accretion energy. In the course of a few million years, however, the accretion rate in the disk drops and this source of midplane heating decays. As a result of this the snow line, which due to the accretional heating may initially be very far out (50 AU or so) will creep inward until it reaches about $1 \mathrm{AU}$ (Davis 2005). Only when the gas and dust dissipate, after 3 to 10 million years, the snow line will move again further out because the disk becomes optically thin, and the dust can no longer keep the midplane cold.

The viscous evolution of a protoplanetary disk involves accretion as well as spreading of the disk. This has been described originally by Lynden-Bell \& Pringle (1974), but it is still believed to be true. However, the mass of the disk decays only very slowly in the later stages of the evolution of the disk. It may take many tens of Myr to render the disk optically thin, while observations show that disks are destroyed in about 3 to 10 Myrs (e.g. Haisch et al. 2001; Carpenter et al. 2005). A possible solution to this problem comes from photoevaporation. The process of photoevaporation of disks by the Extreme UV (EUV) photons of the central star has been described in the context of massive stars by Hollenbach et al. (1994). For lower mass stars, Clarke et al. (2001) showed that EUV photoevaporation will drill a hole in the disk at about a few AU once the evolution of the disk has made the accretion rate to drop below about $\dot{M} \sim$ $10^{-9} M_{\odot} /$ yr. Once this hole is drilled, the disk is quickly destroyed through an insideout evaporation process (Alexander et al. 2006). However, if the initial mass of the disk is large (say, $0.1 M_{\odot}$ ), then it may take quite a while before this point is reached (over $10 \mathrm{Myr}$ ). Again, this appears to be problematic in the light of the observations. Gorti \& Hollenbach (2009) have shown that another kind of UV photons, far-UV (FUV) photons, have a deeper penetration depth in the disk and overall contain more luminosity than the EUV photons. Also these photons tend to attack the outer regions of the disk (50-100 AU or so), which is the location where the mass reservoir of the disk is. This new photoevaporation process is therefore able to remove much more mass per second from the disk than EUV photoevaporation can. The viscous evolution in fact helps this process. As the disk wants to spread, it conveys material outward toward this 50-100 AU evaporation radius, where this material will be removed, leaving room for more material to be conveyed which also gets removed. This means that the combination of outward 
drift of the gas and the photoevaporation by FUV photons is very effective in destroying the disk (Gorti, Dullemond \& Hollenbach in press). It is, however, very hard to model this process correctly. In the Gorti et al. model the gas temperature is calculated in great detail, but the evaporation process that follows is computed using simple analytic formulae. It is clear that the next step is to include the detailed heating/cooling physics into a 2-D axisymmetric hydrodynamics model where the 2-D dynamics of the disk is treated self-consistently, and thus the complex 2-D motion of the gas can be properly followed from the surface of the disk toward the sonic surface. Once such models are made with reasonably reliable detail, the results may be used to predict disk life times, which can be compared to observed life times and which are important ingredients in models of planet formation.

The models of photoevaporation of disks may also provide clues to the mysterious large inner holes observed in a subset of protoplanetary disks. Lately these objects are called "transition disks", as this is inspired by the idea that these disks form the phase between classical T Tauri stars and debris disks. Some authors also call them "cold disks", since the lack of hot dust in these inner holes renders the remaining emission "cold" (long wavelengths). The inference of the existence of these holes is usually done from the lack of cold dust in the spectral energy distribution (SED), but there are some examples where spatially resolved observations seem to confirm this (e.g. TW Hydra, Hughes et al. 2007; Ratzka et al. 2007). There is a subclass of these disks which have indeed such large inner holes, but still a minor amount of hot dust very close to the star. In these objects there is a clear lack of dust at temperatures between $100 \mathrm{~K}$ and $1000 \mathrm{~K}$ or thereabout, but there is hot dust (at about 1000-1500 K, i.e. residing around $0.1 \mathrm{AU}$ around a T Tauri star) and there is cold dust (at temperatures below about $100 \mathrm{~K}$, i.e. beyond about 20 $\mathrm{AU}$ or so). Also here these holes are mainly inferred from the SED, but also here some image evidence has been obtained (Brown et al. 2009).

The inner holes created by EUV photons might be an explanation for some of these sources. But Gorti et al. (in press) have shown that also FUV photoevaporation can drill a hole in the disk, and at much earlier times. So it is unclear which of the two mechanisms of photoevaporation would then be responsible for the observed transition disks.

\section{References}

Alexander, R. D., Clarke, C. C., \& Pringle, J. E. 2006, MNRAS 369, 229

Brown J. M., Blake G. A., Qi, C., Dullemond, C. P., Wilner D. J., \& Williams J. P. 2009, ApJ 704,496

Carpenter, J. M., Wolf, S., Schreyer, K., Launhardt, R., \& Henning, T. 2005, AJ 129, 1049

Chiang, E. I. \& Goldreich, P. 1997, ApJ 490, 368 ApJ 490, 368

Clarke, C. J., Gendrin, A., \& Sotomayor, M. 2001, MNRAS 328, 485

Davis, S. S. 2005, ApJ 620, 994

Gorti, U. \& Hollenbach, D. 2009, ApJ 690, 1539

Haisch, K. E., Lada, E. A., \& Lada, C. J. 2001, ApJ 553:L153.

Hollenbach, D., Johnstone, D., Lizano, S., \& Shu, F. 1994, ApJ 428, 654

Hughes, A. M., Wilner, D. J., Calvet, N., D’Alessio, P., Claussen, M. J., \& Hogerheijde, M. R. 2007, ApJ 664, 536.

Lynden-Bell, D. \& Pringle, J. E. 1974, MNRAS 168, 603

Ratzka, T., Leinert, C., Henning, T., Bouwman, J., Dullemond, C. P., \& Jaffe, W. 2007, A\&A 471,173 